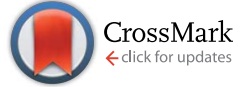

Cite this: RSC Adv., 2016, 6, 73336

Received 2nd July 2016

Accepted 26th July 2016

DOI: 10.1039/c6ra17030g

www.rsc.org/advances

\section{Competent overall water-splitting electrocatalysts derived from ZIF-67 grown on carbon cloth $\uparrow$}

\author{
Xin Liu, ${ }^{\text {ab }}$ Jinmei Dong, ${ }^{\text {ab }}$ Bo You ${ }^{a}$ and Yujie Sun*a
}

The design of nonprecious, bifunctional, and highly competent electrocatalysts for both $\mathrm{H}_{2}$ and $\mathrm{O}_{2}$ evolution reactions (HER and OER) has attracted increasing interest recently. Herein, we report a cobaltbased electrocatalyst derived from ZIF-67 grown on carbon cloth (Co-P/NC/CC) for overall water splitting electrocatalysis. The as-prepared $\mathrm{Co}-\mathrm{P} / \mathrm{NC} / \mathrm{CC}$ catalyst exhibited remarkable catalytic performance in $1 \mathrm{M} \mathrm{KOH}$ with Tafel slopes of 52 and $61 \mathrm{mV} \mathrm{dec}^{-1}$ for HER and OER, respectively. When serving as catalysts for both the cathode and anode, our Co-P/NC/CC demonstrated high efficiency and strong robustness. A thorough comparison with other control samples and detailed characterization results revealed that the superior activity and excellent stability of $\mathrm{Co}-\mathrm{N}-\mathrm{C}$ likely originated from the highly porous, self-supported, and binder-free nature of the electrocatalyst, as well as the high conductivity of carbon cloth. Hence, direct decoration of metal organic frameworks on conductive substrates represents an effective approach for the development of electrocatalysts not only promising for water splitting but also for many other applications.

\section{Introduction}

Along with the increasing global energy demands and deteriorating climate change resulting from fossil fuel utilization, clean and renewable energy-driven water splitting to produce $\mathrm{H}_{2}$ and $\mathrm{O}_{2}$ has been widely considered as a promising approach to alleviate our reliance on fossil fuels and mitigate climate change. ${ }^{1,2}$ The slow kinetics of both $\mathrm{H}_{2}$ and $\mathrm{O}_{2}$ evolution reactions (HER and OER) necessitate the development of competent electrocatalysts., ${ }^{\mathbf{3} 4}$ Even though platinum, iridium- and ruthenium-based oxides have long been recognized as start-ofthe-art electrocatalysts for HER and OER, respectively, their low abundance and thus high cost call for the exploration of alternative earth-abundant catalyst candidates. Indeed, recent years have witnessed the emergence of many excellent HER and OER catalysts composed of low-cost materials, such as $1^{\text {st }}$-row transition metals, ${ }^{5}$ oxides, ${ }^{6}$ oxyhydroxides,${ }^{7-9}$ sulfides,${ }^{10-14}$ selenides, ${ }^{15,16}$ phosphides, ${ }^{17-19}$ nitrides,${ }^{20}$ as well as metal-free electrocatalysts. $^{21}$ The large earth abundance and rich redox properties of these catalysts make them quite promising for large scale employment of electrocatalytic water splitting. ${ }^{22,23}$ In order to accomplish high energy conversion efficiency, water splitting is better to be conducted in either strongly acidic or

${ }^{a}$ Department of Chemistry and Biochemistry, Utah State University, Logan, Utah 84322, USA. E-mail: yujie.sun@usu.edu; Fax: +1-435-797-3390; Tel: +1-435-797-7608 ${ }^{b}$ Key Laboratory of Comprehensive and Highly Efficient Utilization of Salt Lake Resources, Chinese Academy of Sciences, 18 Xinning Road, Xi'ning 810008, China $\dagger$ Electronic supplementary information (ESI) available: Additional electrochemistry and characterization data, Fig. S1-S12 and Tables S1-S3. See DOI: $10.1039 / \mathrm{c} 6 \mathrm{ra} 17030 \mathrm{~g}$ alkaline solution to minimize the electrolyte resistance. ${ }^{24,25}$ Therefore, it requires that the HER and OER catalysts are able to function in the same medium. ${ }^{26}$ Within this context, it might be cost competitive to develop bifunctional electrocatalysts (or catalyst precursors) which are active for both HER and OER in the same electrolyte.

Taking advantage of the large specific surface area and high porosity of metal organic frameworks (MOF), our group recently reported several MOF-derived electrocatalysts for various reactions, such as water splitting and oxygen reduction reaction. ${ }^{27,28}$ We demonstrated that a cobalt imidazolate-based MOF, ZIF-67, was able to act as a bifunctional electrocatalyst for overall water splitting after pyrolysis and phosphidation treatments. ${ }^{27}$ In order to load the ZIF-67-derived catalyst on the working electrode, a conductive polymer, Nafion, was utilized to immobilize the catalyst on the electrode surface. Such a viscous binder of Nafion will inevitably result in buried active sites, decreased specific surface area, and increased interfacial resistance, which would result in inferior performance of the catalyst and complicate the electrode preparation step. ${ }^{29-31}$ Therefore, we seek to develop MOF-derived electrocatalysts directly grown on conductive substrates with intimate physical and electric contact. $^{32}$ To the best of our knowledge, direct growing of MOFderived electrocatalysts on conductive substrates for overall water splitting has received scarce attention.

Herein, we report a facile and effective strategy to anchor ZIF-67 on the surface of carbon cloth (CC, Fig. S1a $\dagger$ ) to yield ZIF-67/CC. Carbon cloth is a type of cheap and commercially available textile with high conductivity, robust corrosion resistance, excellent flexibility, and strong mechanical strength, 
representing an ideal 3D substrate to integrate catalysts for electrocatalytic applications. ${ }^{33-36}$ Pyrolysis of ZIF-67/CC followed by phosphidation resulted in cobalt phosphide nanoparticles imbedded in nitrogen-containing carbon matrices anchored on carbon cloth tightly (Co-P/NC/CC). Electrochemical studies of Co-P/NC/CC demonstrate its excellent activities for electrocatalytic HER, OER, and overall water splitting. A myriad of materials characterization techniques were also employed to study the composition and morphology of Co-P/NC/CC prior to and post electrocatalytic reactions. The excellent electrocatalytic performance of Co-P/NC/CC compared to control samples, such as ZIF-67-derived catalyst particles drop casted on carbon cloth, is primarily attributed to the intimate contact between $\mathrm{Co}-\mathrm{P} / \mathrm{NC}$ catalyst and carbon cloth, as well as the large surface area and high conductivity of the latter.

\section{Experimental}

\subsection{Chemicals}

2-Methylimidazole, potassium hydroxide and sodium hypophosphite monohydrate were purchased from Alfa Aesar. Cobalt nitrate hexahydrate and Nafion 117 solution (5\% in a mixture of lower aliphatic alcohols and water) were purchased from Sigma Aldrich. Methanol was purchased from Decon Laboratories. All chemicals were used as received without any further purification. Carbon cloth was purchased from Fuel Cell Store. Deionized water (18 $\mathrm{M} \Omega \mathrm{cm}$ ) obtained from a Barnstead E-Pure system was used in all experiments.

\subsection{Syntheses}

Synthesis of ZIF-67. ZIF-67 was synthesized according to our published method. ${ }^{27}$ Specifically, $3.6 \mathrm{mmol} \mathrm{Co}\left(\mathrm{NO}_{3}\right)_{2} \cdot 6 \mathrm{H}_{2} \mathrm{O}$ was dissolved in $12 \mathrm{~mL}$ methanol to form a clear solution A. 14.4 mmol 2-methylimidazole was dissolved in $4 \mathrm{~mL}$ methanol to form a clear solution B. Then, solution B was injected into solution A. After thorough mixing at room temperature for $2 \mathrm{~h}$, the as-obtained precipitates were centrifuged and washed with copious amount of methanol to yield ZIF-67.

Synthesis of ZIF-67/CC. $3.6 \mathrm{mmol} \mathrm{Co}\left(\mathrm{NO}_{3}\right)_{2} \cdot 6 \mathrm{H}_{2} \mathrm{O}$ was dissolved in $12 \mathrm{~mL}$ methanol to form a clear solution A. A carbon cloth $(1 \mathrm{~cm} \times 4 \mathrm{~cm})$ was immersed into the solution. $14.4 \mathrm{mmol}$ 2-methylimidazole was dissolved in $4 \mathrm{~mL}$ methanol to form a clear solution B. Subsequently, solution B was injected into solution A. After thorough mixing at room temperature for $2 \mathrm{~h}$, the carbon cloth anchored with ZIF-67 was retrieved from the solution and washed with methanol thoroughly, resulting in ZIF-67/CC.

Syntheses of Co/NC/CC and Co-P/NC/CC. ZIF-67/CC was placed in a tube furnace and heated to $900{ }^{\circ} \mathrm{C}$ for $3 \mathrm{~h}$ under flowing argon to obtain $\mathrm{Co} / \mathrm{NC} / \mathrm{CC}$. Subsequently, one piece of $\mathrm{Co} / \mathrm{NC} / \mathrm{CC}$ and $1.0 \mathrm{~g}$ sodium hypophosphite monohydrate were heated at $300{ }^{\circ} \mathrm{C}$ for $2 \mathrm{~h}$ under flowing argon with sodium hypophosphite located at the upstream site, resulting in the Co$\mathrm{P} / \mathrm{NC} / \mathrm{CC}$.

Synthesis of Co-P/NC-CC. Co-P/NC was synthesized according to our published method. ZIF-67 was carburized and phosphatized following the same conditions discussed above to yield Co-P/NC. Subsequently, Co-P/NC was drop-casted on carbon cloth with the same mass loading as that of $\mathrm{Co}-\mathrm{P} / \mathrm{NC} /$ CC. In a typical procedure, $10.3 \mathrm{mg} \mathrm{Co}-\mathrm{P} / \mathrm{NC}$ was dispersed in a mixture solution of $30 \mu \mathrm{L} 5 \%$ Nafion, $250 \mu \mathrm{L}$ ethanol, and 720 $\mu \mathrm{L}$ water. The mixture was sonicated in an ice bath for $30 \mathrm{~min}$ to obtain a homogeneous catalyst ink. Next, $10 \mu \mathrm{L}$ catalyst ink was loaded onto a carbon cloth with a loading amount of $0.206 \mathrm{mg} \mathrm{cm}^{-2}$.

\subsection{Physical methods}

Scanning electron microscopy (SEM) and elemental mapping analysis were conducted on a FEI QUANTA FEG 650 (FEI, USA). $\mathrm{X}$-ray diffraction (XRD) data were recorded on a Rigaku MinifexII Desktop X-ray diffractometer. The cobalt and phosphorus quantities of each catalyst sample were analysed by a Thermo Electron iCAP inductively coupled plasma atomic emission spectrophotometer (ICP-AES). X-ray photoelectron spectra were collected on a Kratos Axis Ultra instrument (Chestnut Ridge, NY). The catalyst samples were affixed on a stainless steel Kratos sample bar, loaded into the instrument's load lock chamber, and evacuated to $5 \times 10^{-8}$ Torr before they were transferred into the sample analysis chamber under ultrahigh vacuum conditions $\left(\sim 10^{-10}\right.$ Torr). X-ray photoelectron spectra were taken using the monochromatic $\mathrm{Al} \mathrm{K} \alpha$ source $(1486.7 \mathrm{eV})$ at a $300 \times 700 \mu \mathrm{m}$ spot size. High resolution regions at the binding energy of interest were taken for each sample. The samples were also sputter cleaned inside the analysis chamber with $1 \mathrm{keV} \mathrm{Ar}^{+}$ions for 30 seconds to remove adventitious contaminants. The XPS data were analysed using CASA XPS software and energy corrections on high resolution scans were calibrated by referencing the $\mathrm{C} 1 \mathrm{~s}$ peak of adventitious carbon to $284.5 \mathrm{eV}$.

\subsection{Electrocatalytic measurements}

Linear sweep voltammetry (LSV) was performed by a computercontrolled Gamry Interface 1000 electrochemical workstation with a three-electrode configuration. The catalyst-coated carbon cloth was directly used as the working electrode. A $\mathrm{Ag} / \mathrm{AgCl}$ (sat. $\mathrm{KCl}$ ) electrode was used as the reference electrode and a Pt wire as the counter electrode. All potentials reported herein were referenced to the reversible hydrogen electrode (RHE) through calibration. 1.0 M KOH was utilized. Electric impedance spectroscopy was carried out in the same configuration from $10^{5}$ to $0.1 \mathrm{~Hz}$ with an AC potential amplitude of 30 $\mathrm{mV}$. The electrochemical double-layer capacitance $\left(C_{\mathrm{dl}}\right)$ of each sample was evaluated via cyclic voltammetry in a non-faradaic region from 0.123 to $0.223 \mathrm{~V} v$ s. RHE for HER and 1.223 to 1.323 $\mathrm{V} v s$. RHE for OER at scan rates ranging from 4 to $40 \mathrm{mV} \mathrm{s}^{-1}$. The resulting linear slope is twice of the $C_{\mathrm{dl}}$. For overall water splitting tests, the same-type catalyst-coated carbon cloth electrodes were used for anode and cathode directly. The final chronopotentiometry was conducted at a current density of $10 \mathrm{~mA} \mathrm{~cm}{ }^{-2}$ for $20 \mathrm{~h}$. 


\section{Results and discussion}

The overall synthetic route is illustrated in Scheme 1. In the presence of carbon cloth, a mixture of cobalt nitrate and 2methylimidazole in methanol resulted in ZIF-67 preferentially grown on this conductive substrate, probably due to ample nucleation sites on the surface of carbon cloth. The as-prepared ZIF-67 anchored on carbon cloth was named as ZIF-67/CC. Fig. 1a and b presents the SEM images of ZIF-67, showing regular nano-polyhedrons grown on carbon cloth tightly. The size of these ZIF-67 polyhedrons is around $0.5-1 \mu \mathrm{m}$, close to reported samples. ${ }^{27}$ Element mapping analysis (Fig. 1c) suggested that ZIF-67 mainly consisted of $\mathrm{Co}, \mathrm{C}$, and N, all of which were homogeneously distributed throughout the entire nanopolyhedrons.

Subsequently, ZIF-67/CC was carburized under argon at 900 ${ }^{\circ} \mathrm{C}$ for $3 \mathrm{~h}$. During this process, ZIF-67 nano-polyhedrons transformed to metallic cobalt nanoparticles imbedded in nitrogen-containing carbon matrices, which was denoted as $\mathrm{Co} /$ NC/CC. The SEM image of Co/NC/CC (Fig. S1b $\dagger$ ) indicated that the original regular and smooth nano-polyhedrons of ZIF-67 shrank to rough porous composites. Nevertheless, the discrete nanopolyhedrons could still be identified. Next, Co/NC/CC was phosphatized under argon at $300{ }^{\circ} \mathrm{C}$ for $2 \mathrm{~h}$, utilizing sodium hypophosphite as the phosphorous source and resulting in cobalt phosphide nanoparticles decorated within nitrogencontaining carbon matrices. This final product was named as Co-P/NC/CC. As shown in Fig. 1d, the SEM image of Co-P/NC/ CC proved that the integrity of each shrunk nano-polyhedron was preserved, although a close inspection (Fig. 1e) implied some agglomeration. It should be noted that these new nanopolyhedrons were still tightly anchored on the surface of carbon cloth. The large enrichment of phosphorous in these nano-polyhedrons was well manifested in the elemental mapping images shown in Fig. 1f, along with the homogeneous distribution of $\mathrm{Co}, \mathrm{P}, \mathrm{N}$, and $\mathrm{C}$.

The XRD pattern of ZIF-67/CC (Fig. S2 $\dagger$ ) confirmed the crystallinity and identity of ZIF-67 grown on carbon cloth. ${ }^{37,38}$ After pyrolysis, the weak and broad XRD peaks of $\mathrm{Co} / \mathrm{NC} / \mathrm{CC}$

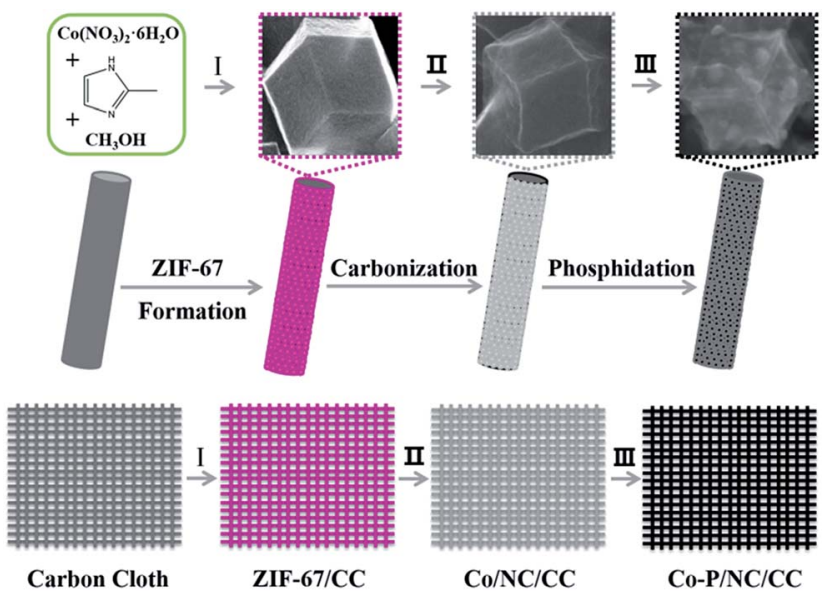

Scheme 1 Synthetic procedure of Co-P/NC/CC.
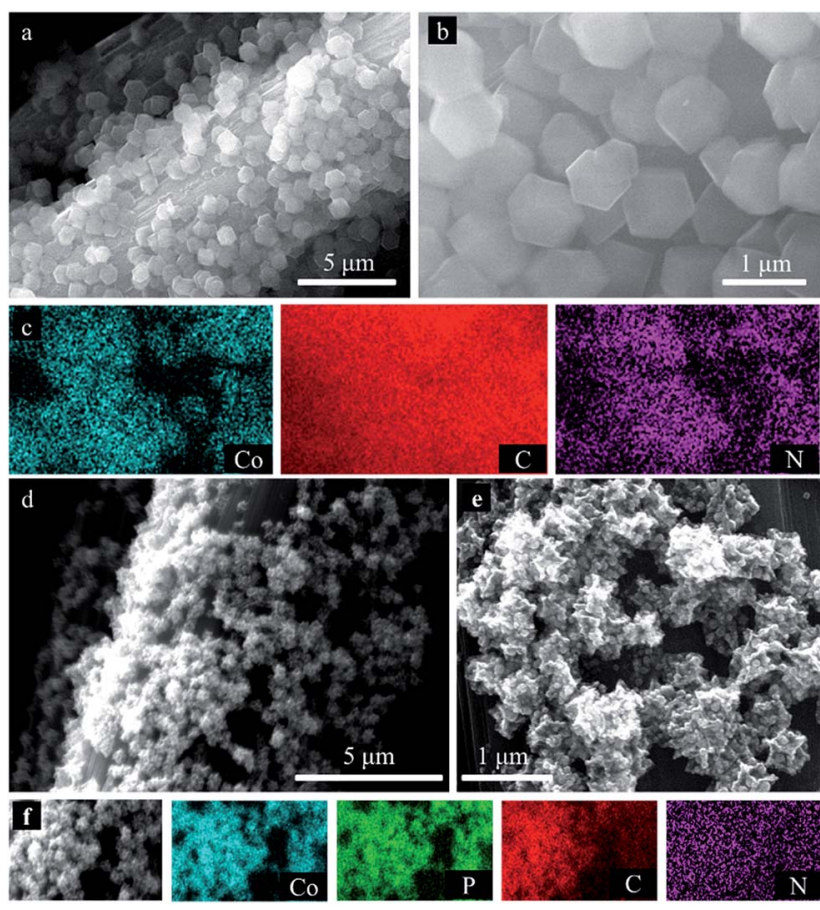

Fig. 1 ( $a$ and b) SEM images of ZIF-67/CC and (c) the elemental mapping images of ZIF-67/CC showing the distributions of $\mathrm{Co}, \mathrm{C}$, and $\mathrm{N}$. (d and e) SEM images of Co-P/NC/CC and (f) the elemental mapping images of $\mathrm{Co}-\mathrm{P} / \mathrm{NC} / \mathrm{CC}$ showing the distributions of $\mathrm{Co}, \mathrm{P}$, $\mathrm{C}$, and $\mathrm{N}$.

(Fig. S3 $\dagger$ ) at $44.2^{\circ}$ and $51.5^{\circ}$ suggested the formation of metallic cobalt; while the relatively intense peak around $25-30^{\circ}$ was due to the substrate of carbon cloth. After phosphidation, despite the lack of well-resolved XRD peaks for Co-P/NC/CC, a comparison with standard XRD patterns of $\mathrm{CoP}$ and $\mathrm{Co}_{2} \mathrm{P}$ suggested the formation of a mixture of $\mathrm{CoP}$ and $\mathrm{Co}_{2} \mathrm{P}$ in $\mathrm{Co}-\mathrm{P} /$ NC/CC (Fig. S4 $\dagger$ ). Elemental analysis data listed in Table S1 $\dagger$ presented a $\mathrm{Co} / \mathrm{P}$ atomic ratio is close to 4.5 (much larger than 2), implying that only a layer of $\mathrm{CoP}$ and $\mathrm{Co}_{2} \mathrm{P}$ mixture was formed on the surface while the inner composite was still metallic cobalt in Co-P/NC/CC.

In order to further shed light on the composition and valence states of the elements in each catalyst sample, X-ray photoelectron spectroscopy (XPS) was collected. Fig. S5a $\uparrow$ presents the survey spectrum of $\mathrm{Co}-\mathrm{P} / \mathrm{NC} / \mathrm{CC}$ which showed all the anticipated elements of Co, P, C, and N, consistent with the elemental mapping results (Fig. 1f). The high-resolution Co 2p XPS spectrum of Co-P/NC/CC is plotted in Fig. 2a. Deconvolution of this Co $2 \mathrm{p}$ spectrum resulted in primary Co $2 \mathrm{p}_{3 / 2}$ peaks at 778.5 and $782.1 \mathrm{eV}$, corresponding to cobalt phosphides and cobalt oxides, respectively. The presence of cobalt oxides was probably due to oxidation in air prior to the XPS measurement. ${ }^{33,39}$ The weak feature around $793.6 \mathrm{eV}$ could be assigned to the Co $2 \mathrm{p}_{1 / 2}$ binding energy. The deconvolution of the high-resolution $\mathrm{P} 2 \mathrm{p}$ XPS spectrum led to a prominent peak at $134.0 \mathrm{eV}$, attributable to oxidized phosphorous species (e.g., phosphate). In the meantime, typical phosphide signal could still be observed at 129.7 and $131.1 \mathrm{eV}$, which were attributed to the $\mathrm{P} 2 \mathrm{p}_{3 / 2}$ and $2 \mathrm{p}_{1 /}$ 

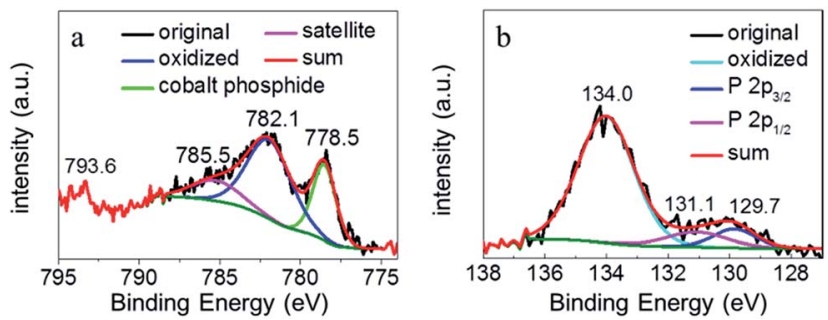

Fig. 2 High-resolution of XPS spectra of Co-P/NC/CC in the (a) Co $2 p$ region and (b) $P 2 p$ region.

${ }_{2}$ features of phosphorous in cobalt phosphide. Overall, the binding energies at 778.5 and $129.7 \mathrm{eV}$ were quite close to those of CoP. ${ }^{40}$ In addition, the high-resolution N 2p XPS spectrum was also collected and deconvoluted in Fig. S5b, $\uparrow$ displaying binding energies at approximately 398.9, 399.4, and $401.7 \mathrm{eV}$, which could be attributed to pyridinic N, Co-N, and graphitic N, respectively.

With the detailed characterization data in hand, we next assessed the electrocatalytic performance of these catalysts for $\mathrm{H}_{2}$ evolution. All the electrochemical experiments were conducted in $1.0 \mathrm{M} \mathrm{KOH}$ using a three-electrode configuration unless otherwise noted. The linear sweep voltammetry (LSV) curves of $\mathrm{Co}-\mathrm{P} / \mathrm{NC} / \mathrm{CC}$ and control samples (carbon cloth, ZIF$67 / \mathrm{CC}$, and $\mathrm{Co} / \mathrm{NC} / \mathrm{CC}$ ) were plotted in Fig. 3a. It is apparent that $\mathrm{Co}-\mathrm{P} / \mathrm{NC} / \mathrm{CC}$ catalyzed $\mathrm{H}_{2}$ evolution much earlier than all the control samples with a catalytic onset potential around -0.1 $\mathrm{V} v s$. reversible hydrogen electrode (RHE). Without phosphidation, $\mathrm{Co} / \mathrm{NC} / \mathrm{CC}$ initiated HER catalytic current approximately $50 \mathrm{mV}$ more negative than that of Co-P/NC/CC. Most likely due to the poor conductivity and lack of active sites in ZIF-67, ZIF-67/
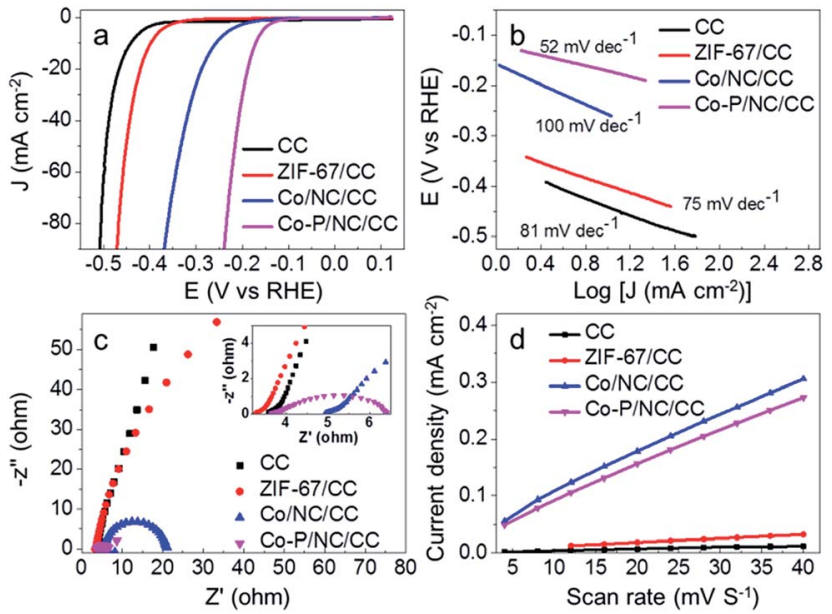

Fig. 3 (a) LSV curves of Co-P/NC/CC (purple), Co/NC/CC (blue), ZIF$67 / \mathrm{CC}$ (red), and CC (black) for HER in $1.0 \mathrm{M} \mathrm{KOH}$ at a scan rate of $2 \mathrm{mV}$ $\mathrm{s}^{-1}$. (b) Tafel plots of Co-P/NC/CC (purple), Co/NC/CC (blue), ZIF-67/ CC (red), and CC (black) for HER. (c) Nyquist plots of Co-P/NC/CC (purple), Co/NC/CC (blue), ZIF-67/CC (red), and CC (black) measured at $-247 \mathrm{mV}$ vs. RHE. Inset shows the expanded region around the intercepts at the $x$ axis. (d) Scan rate dependence of the current densities of Co-P/NC/CC (purple), Co/NC/CC (blue), ZIF-67/CC (red), and $\mathrm{CC}$ (black) at $0.173 \mathrm{~V}$ vs. RHE.
CC only acted slightly better than the background carbon cloth for HER. Specifically, Co-P/NC/CC only required overpotentials of -171 and $-243 \mathrm{mV}$ to reach catalytic current densities of 10 and $100 \mathrm{~mA} \mathrm{~cm}{ }^{-2}$, respectively. These overpotential requirements are much smaller than those of $\mathrm{Co} / \mathrm{NC} / \mathrm{CC}$ and ZIF-67/CC to achieve the same current densities and also compare quite favourably with those of recently reported HER electrocatalysts in strongly alkaline electrolytes (see detailed comparison in Table S2 $\dagger$ ). ${ }^{41-44}$ Even though Pt/C exhibited a smaller onset potential, the current density of our $\mathrm{Co}-\mathrm{P} / \mathrm{NC} / \mathrm{CC}$ surpassed that of $\mathrm{Pt} / \mathrm{C}$ beyond $-220 \mathrm{mV} v$ s. RHE (Fig. S8a $\dagger$ ). Fig. 3b presented the derived Tafel plots of each catalyst and apparently $\mathrm{Co}-\mathrm{P} / \mathrm{NC} /$ CC exhibited a much smaller Tafel slope $\left(52 \mathrm{mV} \mathrm{dec}^{-1}\right)$ compared to those of carbon cloth $\left(81 \mathrm{mV} \mathrm{dec}^{-1}\right)$, ZIF-67/CC (75 $\left.\mathrm{mV} \mathrm{dec}^{-1}\right)$, and $\mathrm{Co} / \mathrm{NC} / \mathrm{CC}\left(100 \mathrm{mV} \mathrm{dec}^{-1}\right)$, implying a faster HER kinetic rate of the former. In addition, electric impedance spectroscopy was collected to further probe the superior HER activity of Co-P/NC/CC. As shown in Fig. 3c, the Nyquist plot of $\mathrm{Co}-\mathrm{P} / \mathrm{NC} / \mathrm{CC}$ showed a smaller semi-circular diameter than those of the control samples and thus demonstrated a lower resistance for electrocatalytic HER. In order to determine whether the high HER performance of $\mathrm{Co}-\mathrm{P} / \mathrm{NC} / \mathrm{CC}$ is due to its increased electrochemically active surface area (ECSA), we next measured its double-layer capacitance via cyclic voltammetry in the non-faradaic region ( 0.123 to $0.223 \mathrm{~V} v$ s. RHE, Fig. S6†). It has been widely accepted that cyclic voltammetry at nonfaradaic potentials is able to estimate ECSA, especially when the electrodes consist of similar composites. By plotting the difference in current density between the anodic and cathodic scans at $0.173 \mathrm{~V} v$ s. RHE, nearly linear plots could be obtained in Fig. 3d. The extracted slopes of the linear fitting resulted in similar ECSA for both $\mathrm{Co}-\mathrm{P} / \mathrm{NC} / \mathrm{CC}$ and $\mathrm{Co} / \mathrm{NC} / \mathrm{CC}$, which were substantially larger than those of ZIF-67/CC and carbon cloth. Therefore, it is clear that although large ECSA is beneficial towards HER, the higher HER activity of Co-P/NC/CC relative to $\mathrm{Co} / \mathrm{NC} / \mathrm{CC}$ is most likely owning to the phosphidation step which yielded more HER active cobalt phosphides on the cobalt nanoparticles.

The OER performances of these catalysts were also evaluated in the same electrolyte, $1.0 \mathrm{M} \mathrm{KOH}$. Apparently, Co-P/NC/CC exhibited a much smaller onset potential of $\sim 1.5 \mathrm{~V} v s$. RHE and greater catalytic current than those of $\mathrm{Co} / \mathrm{NC} / \mathrm{CC}$, ZIF-67/ $\mathrm{CC}$, and CC (Fig. 4a). Noticeably, Co/NC/CC required an overpotential of $360 \mathrm{mV}$ to reach a current density of $10 \mathrm{~mA} \mathrm{~cm}{ }^{-2}$; while after phosphidation, $\mathrm{Co}-\mathrm{P} / \mathrm{NC} / \mathrm{CC}$ only required an overpotential of $330 \mathrm{mV}$ to arrive at the same current density. ZIF-67/ $\mathrm{CC}$ and carbon cloth were not very active towards OER and they required potential more positive than $1.6 \mathrm{~V}$ to produce appreciable catalytic current. Even though these catalysts exhibited substantially different catalytic onsets, their derived Tafel slopes fell in a close range of $50-70 \mathrm{mV} \mathrm{dec}{ }^{-1}$. Electric impedance spectra of these catalysts are shown in Fig. 4c, wherein Co$\mathrm{P} / \mathrm{NC} / \mathrm{CC}$ presented the smallest resistance, in agreement with its best OER activity. Despite the similarity in the electrochemical surface areas of $\mathrm{Co}-\mathrm{P} / \mathrm{NC} / \mathrm{CC}$ and $\mathrm{Co} / \mathrm{NC} / \mathrm{CC}$ for HER application, a rather obvious enhancement in ECSA was observed for $\mathrm{Co}-\mathrm{P} / \mathrm{NC} / \mathrm{CC}$ relative to $\mathrm{Co} / \mathrm{NC} / \mathrm{CC}$ for $\mathrm{O}_{2}$ evolution 

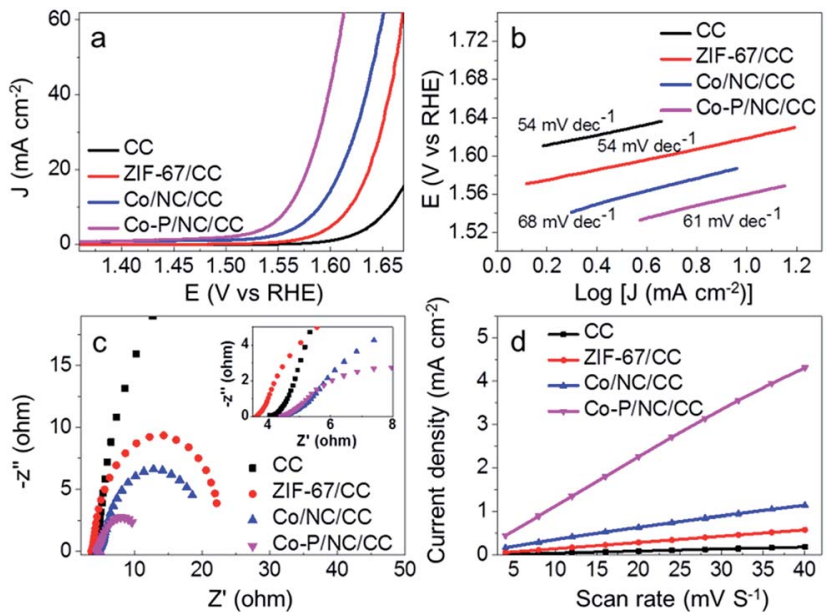

Fig. 4 (a) LSV curves of Co-P/NC/CC (purple), Co/NC/CC (blue), ZIF$67 / \mathrm{CC}$ (red), and CC (black) for OER in $1.0 \mathrm{M} \mathrm{KOH}$ at a scan rate of $2 \mathrm{mV}$ $\mathrm{s}^{-1}$. (b) Tafel plots of Co-P/NC/CC (purple), Co/NC/CC (blue), ZIF-67/ CC (red), and CC (black) for OER. (c) EIS curves of Co-P/NC/CC (purple), Co/NC/CC (blue), ZIF-67/CC (red), and CC (black) measured at $1.573 \mathrm{~V}$ vs. RHE. Inset shows the expanded region around the intercepts at the $x$ axis. (d) Scan rate dependence of the current densities of Co-P/NC/CC (purple), Co/NC/CC (blue), ZIF-67/CC (red), and $\mathrm{CC}$ (black) at $1.273 \mathrm{~V}$ vs. RHE.

(Fig. 4d and S7 $\dagger$ ). It implied that during electrocatalytic OER, the surface oxidation of Co-P nanoparticles would likely result in more porous and hence a larger number of electrochemically active sites than what occurred in the case of $\mathrm{Co} / \mathrm{NC} / \mathrm{CC}$. This observation is consistent with recent reports showing that metal phosphide-based electrocatalysts actually demonstrated better OER performance the corresponding metal oxides. The estimated ECSA values of Co-P/NC/CC, Co/NC/CC, ZIF-67/CC, and carbon cloth follow the same trend of their OER activity, emphasizing the critical role that ECSA plays in electrocatalysis. A detailed comparison of $\mathrm{Co}-\mathrm{P} / \mathrm{NC} / \mathrm{CC}$ with other reported electrocatalysts for OER under strongly alkaline conditions can be found in Table S3, $\dagger$ suggesting the excellent performance of Co-P/NC/CC for OER applications. ${ }^{45-49}$ Noticeably, our Co-P/ $\mathrm{NC} / \mathrm{CC}$ could also rival the state-of-the-art OER catalyst $\mathrm{IrO}_{2}$ (Fig. S8b†), showing similar onset potential but much faster catalytic current rise.

In order to demonstrate the fact that directly growing ZIF-67 on carbon cloth is crucial towards the excellent electrocatalytic performance of $\mathrm{Co}-\mathrm{P} / \mathrm{NC} / \mathrm{CC}$ for both HER and OER, we conducted the following control experiments. ZIF-67 nanopolyhedrons were carbonized and phosphatized in a similar fashion as that of ZIF-67/CC. Subsequently, the resulting cobalt phosphide(s)-imbedded and nitrogen containing carbon matrices were loaded on a carbon cloth with Nafion as the polymer binder to produce a control sample Co-P/NC-CC. As shown in Fig. S9, $\dagger$ the HER and OER performances of Co-P/NC$\mathrm{CC}$ are inferior to that of $\mathrm{Co}-\mathrm{P} / \mathrm{NC} / \mathrm{CC}$ with same mass loading of cobalt. The catalytic current of Co-P/NC-CC did not take off until scanning to $-0.3 \mathrm{~V} v s$. RHE for HER and $1.6 \mathrm{~V} v s$. RHE for OER, both of which required a larger overpotential compared to $\mathrm{Co}-\mathrm{P} / \mathrm{NC} / \mathrm{CC}$. In addition, the stability of our $\mathrm{Co}-\mathrm{P} / \mathrm{NC} / \mathrm{CC}$ relative to CC for OER was also inspected. As shown in Fig. S10, $\dagger$ although CC was not stable for long-term OER electrolysis, our $\mathrm{Co}-\mathrm{P} / \mathrm{NC} / \mathrm{CC}$ manifested its perfect stability in 1.0 $\mathrm{M} \mathrm{KOH}$ for $50 \mathrm{~h}$ at $10 \mathrm{~mA} \mathrm{~cm}{ }^{-2}$, showing unchanged chronopotentiometric curve over the entire course of OER electrolysis. These results unambiguously prove the effectiveness of anchoring ZIF-67 on carbon cloth during the preparation stage in producing more active and robust electrocatalysts.

With the excellent HER and OER performance of Co-P/NC/ CC discussed above, we subsequently sought to fabricate a two-electrode electrolyzer for overall water splitting with same type catalyst-loaded carbon cloth electrodes serving as both cathode and anode in 1.0 M KOH. The LSV curves of these twoelectrode systems were compared in Fig. 5a. The Co-P/NC/CC catalyst couple exhibited a catalytic onset potential less than 1.7 V. In fact, this catalyst couple approached current densities of 10 and $100 \mathrm{mV} \mathrm{cm}^{-2}$ at cell voltages of 1.77 and $1.93 \mathrm{~V}$, respectively. In sharp contrast, the control samples of $\mathrm{Co} / \mathrm{NC} /$ CC, Co-P/NC-CC, and ZIF-67/CC required a cell voltage of $1.89,1.95$, and $2.03 \mathrm{~V}$, respectively, to arrive at $10 \mathrm{~mA} \mathrm{~cm}^{-2}$. As expected, carbon cloth was least active for water splitting. $20 \mathrm{~h}$ chronopotentiometry experiments were conducted to assess the stability of these catalyst couples for extended function at a catalytic current of $10 \mathrm{~mA} \mathrm{~cm} \mathrm{~cm}^{-2}$ (Fig. 5b). The Co-P/NC/CC catalyst couple required a voltage of $\sim 1.85 \mathrm{~V}$ and maintained
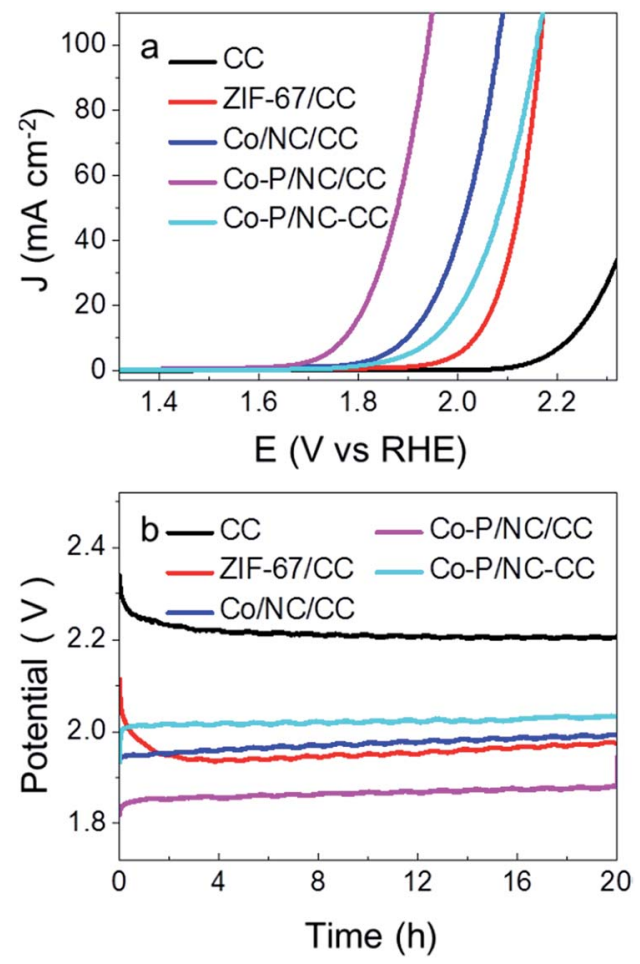

Fig. 5 (a) LSV curves of Co-P/NC/CC (purple), Co/NC/CC (blue), CoP/NC-CC (green), ZIF-67/CC (red), and CC (black) served as both cathode and anode in a two-electrode configuration at a scan rate of 2 $\mathrm{mV} \mathrm{s}^{-1}$ in $1.0 \mathrm{M} \mathrm{KOH}$ for overall water splitting. (b) Chronopotentiometric curves of Co-P/NC/CC (purple), Co/NC/CC (blue), Co-P/NC$\mathrm{CC}$ (green), ZIF-67/CC (red), and CC (black) in $1.0 \mathrm{M} \mathrm{KOH}$ at a current density of $10 \mathrm{~mA} \mathrm{~cm}^{-2}$ for $20 \mathrm{~h}$. 
this voltage throughout the entire course of electrolysis. All the other catalyst samples needed voltages at least $100 \mathrm{mV}$ larger than that of Co-P/NC/CC. After the long-term chronoamperometry experiment, the composition and morphology of $\mathrm{Co}-\mathrm{P} / \mathrm{NC} / \mathrm{CC}$ as cathode (Fig. S11 $\dagger$ ) and anode (Fig. S12 $\dagger$ ) investigated via SEM and elemental mapping analysis. Overall, the anchored cobalt phosphide(s)-based polyhedrons were still observed on the surface of carbon cloth with the anticipated distribution of Co, P, N, and C. A significant amount of oxygen was observed when Co-P/NC/CC acted as the anode, in agreement with the partial oxidation of cobalt phosphide(s)/cobalt during electrocatalytic OER.

\section{Conclusions}

In summary, we described a facile route to synthesize selfsupported and MOF-derived Co-P/NC/CC on carbon cloth as an effective electrocatalyst for overall water splitting. The asprepared $\mathrm{Co}-\mathrm{P} / \mathrm{NC} / \mathrm{CC}$ exhibited remarkable catalytic performance for both HER and OER under alkaline conditions with

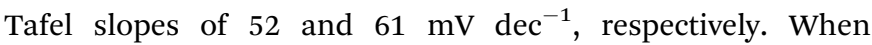
employed as both anode and cathode for overall water splitting test, $\mathrm{Co}-\mathrm{P} / \mathrm{NC} / \mathrm{CC}$ exhibited promising activity and robustness. Compared with other control samples, including ZIF-67/CC, Co/ $\mathrm{NC} / \mathrm{CC}$, and $\mathrm{Co}-\mathrm{P} / \mathrm{NC}-\mathrm{CC}$, the excellent electrocatalytic activity of $\mathrm{Co}-\mathrm{P} / \mathrm{NC} / \mathrm{CC}$ is mainly due to the competent active site (Co$\mathrm{P})$, highly porous and conductive substrate (NC and $\mathrm{CC}$ ), and more importantly the intimate anchoring of $\mathrm{Co}-\mathrm{P} / \mathrm{NC}$ on carbon cloth. It is anticipated that such a convenient direct growing of MOF-derived catalysts on conductive substrate will open a new avenue for the development of promising electroactive materials for various applications.

\section{Acknowledgements}

This work was supported by Utah State University (USU). X. L. acknowledges the financial support from the Chinese Academy of Sciences Funded Overseas Study Program. X. L. and J. D. thank the support of Qinghai Institute of Salt Lakes, Chinese Academy of Sciences. Y. S. also acknowledges the Microscopy Core Facility at USU for the SEM work.

\section{Notes and references}

1 D. Nocera, Chem. Soc. Rev., 2009, 38, 13.

2 H. Gray, Nat. Chem., 2009, $1,7$.

3 M. Shalom, D. Ressnig, X. Yang, G. Clavel, T. Fellinger and M. Antonietti, J. Mater. Chem. A, 2015, 3, 8171.

4 D. Voiry, H. Yamaguchi, J. Li, R. Silva, D. Alves, T. Fujita, M. Chen, T. Asefa, V. Shenoy, G. Eda and M. Chhowalla, Nat. Mater., 2013, 12, 850.

5 M. Gong, W. Zhou, M. Kenney, R. Kapusta, S. Cowley, Y. Wu, B. Lu, M. Lin, D. Wang, J. Yang, B. Hwang and H. Dai, Angew. Chem., Int. Ed., 2015, 127, 12157.

6 Y. Jin, H. Wang, J. Li, X. Yue, Y. Han, P. Shen and Y. Cui, Adv. Mater., 2016, 28, 3785.
7 L. Trotochaud, S. Young, J. Ranney and S. Boettcher, J. Am. Chem. Soc., 2014, 136, 6744.

8 J. Chen, L. Dang, H. Liang, W. Bi, J. Gerken, S. Jin, E. Alp and S. Stahl, J. Am. Chem. Soc., 2015, 137, 15090.

9 O. Diaz-Morales, D. Ferrus-Suspedra and M. Koper, Chem. Sci., 2016, 7, 2639.

10 B. You, N. Jiang, M. Sheng and Y. Sun, Chem. Commun., 2015, 51, 4252.

11 N. Jiang, L. Bogoev, M. Popova, S. Gul, J. Yano and Y. Sun, J. Mater. Chem. A, 2014, 2, 19407.

12 N. Jiang, Q. Tang, M. Sheng, B. You, D.-E. Jiang and Y. Sun, Catal. Sci. Technol., 2016, 6, 1077.

13 B. You, N. Jiang and Y. Sun, Inorg. Chem. Front., 2016, 3, 279. 14 B. You and Y. Sun, Adv. Energy Mater., 2016, 6, 1502333.

15 A. Carim, F. Saadi, M. Soriaga and N. Lewis, J. Mater. Chem. A, 2014, 2, 13835.

16 Y. Zheng, M. Gao, Z. Yu, Q. Gao, H. Gao and S. Yu, Chem. Sci., 2015, 6, 4594.

17 B. You, N. Jiang, M. Sheng, M. Bhushan and Y. Sun, ACS Catal., 2015, 5, 714.

18 J. Ryu, N. Jung, J. Jang, H. Kim and S. Yoo, ACS Catal., 2015, 5, 4066.

19 N. Jiang, B. You, M. Sheng and Y. Sun, ChemCatChem, 2016, 8, 106-112.

20 K. Xu, P. Chen, X. Li, Y. Tong, H. Ding, X. Wu, W. Chu, Z. Peng, C. Wu and Y. Xie, J. Am. Chem. Soc., 2015, 137, 4119.

21 T. Ma, S. Dai, M. Jaroniec and S. Qiao, Angew. Chem., Int. Ed., 2014, 53, 7281.

22 W. Zhou and L. Guo, Chem. Soc. Rev., 2015, 44, 6697.

23 Y. Liu, H. Cheng, M. Lyu, S. Fan, Q. Liu, W. Zhang, Y. Zhi, C. Wang, C. Xiao, S. Wei, B. Ye and Y. Xie, J. Am. Chem. Soc., 2014, 136, 15670.

24 E. Hernández-Pagán, N. Vargas-Barbosa, T. Wang, Y. Zhao, E. Smotkin and T. Mallouk, Energy Environ. Sci., 2012, 5, 7582 .

25 C. Tang, N. Cheng, Z. Pu, W. Xing and X. Sun, Angew. Chem., Int. Ed., 2015, 54, 9351.

26 H. Wang, H. Lee, Y. Deng, Z. Lu, P. Hsu, Y. Liu, D. Lin and Y. Cui, Nat. Commun., 2015, 6, 7261.

27 B. You, N. Jiang, M. Sheng, S. Gul, J. Yano and Y. Sun, Chem. Mater., 2015, 27, 7636.

28 B. You, N. Jiang, M. Sheng, W. Drisdell, J. Yano and Y. Sun, ACS Catal., 2015, 5, 7068.

29 Y. Luo, J. Jiang, W. Zhou, H. Yang, J. Luo, X. Qi, H. Zhang, D. Yu, C. Li and T. Yu, J. Mater. Chem., 2012, 22, 8634.

30 J. Roy-Mayhew, G. Boschloo, A. Hagfeldt and I. Aksay, ACS Appl. Mater. Interfaces, 2012, 4, 2794.

31 N. Cheng, Q. Liu, J. Tian, Y. Xue, A. Asiri, H. Jiang, Y. He and X. Sun, Chem. Commun., 2015, 51, 1616.

32 Z. Zhao, H. Wu, H. He, X. Xu and Y. Jin, Adv. Funct. Mater., 2014, 24, 4698.

33 J. Tian, Q. Liu, A. Asiri and X. Sun, J. Am. Chem. Soc., 2014, 136, 7587.

34 J. Wang, H. Zhong, Z. Wang, F. Meng and X. Zhang, ACS Nano, 2016, 10, 2342.

35 P. Wang, F. Song, R. Amal, Y. Ng and X. Hu, ChemSusChem, 2016, 9, 472 . 
36 W. Fang, D. Liu, Q. Lu, X. Sun and A. Asiri, Electrochem. Commun., 2016, 63, 60.

37 W. Zhang, Z. Wu, H. Jiang and S. Yu, J. Am. Chem. Soc., 2014, 136, 14385.

38 J. Yang, F. Zhang, H. Lu, X. Hong, H. Jiang, Y. Wu and Y. Li, Angew. Chem., Int. Ed., 2015, 54, 10889.

39 H. Li, P. Yang, D. Chu and H. Li, Appl. Catal., A, 2007, 325, 34.

40 A. Grosvenor, S. Wik, R. Cavell and A. Mar, Inorg. Chem., 2005, 44, 8988.

41 H. Zhang, Z. Ma, J. Duan, H. Liu, G. Liu, T. Wang, K. Chang, M. Li, L. Shi, X. Meng, K. Wu and J. Ye, ACS Nano, 2016, 10, 684.
42 H. Jin, J. Wang, D. Su, Z. Wei, Z. Pang and Y. Wang, J. Am. Chem. Soc., 2015, 137, 2688.

43 H. Vrubel and X. Hu, Angew. Chem., Int. Ed., 2012, 51, 12703. 44 L. Feng, H. Vrubel, M. Bensimon and X. Hu, Phys. Chem. Chem. Phys., 2014, 16, 5917.

45 C. McCrory, S. Jung, I. Ferrer, S. Chatman, J. Peters and T. Jaramillo, J. Am. Chem. Soc., 2015, 137, 4347.

46 N. Jiang, B. You, M. Sheng and Y. Sun, Angew. Chem., Int. Ed., 2015, 54, 6251.

47 Y. Li, P. Hasin and Y. Wu, Adv. Mater., 2010, 22, 1926.

48 J. Ren, M. Antonietti and T. Fellinger, Adv. Energy Mater., 2015, 5, 1401660.

49 F. Song and X. Hu, Nat. Commun., 2014, 5, 4477. 